\title{
Relation between Nanoscale Structure of Asphaltene Aggregates and their Macroscopic Solution Properties
}

\author{
L. Barré', J. Jestin ', A. Morisset', T. Palermo' and S. Simon ${ }^{3}$ \\ 1 Institut français du pétrole, IFP, 1-4 av. de Bois-Préau, 92852 Rueil-Malmaison Cedex - France \\ 2 Laboratoire Léon Brillouin, CEA Saclay, 91191 Gif-sur-Yvette Cedex - France \\ 3 Ugelstad Laboratory, Department of Chemical Engineering, the Norwegian University of Science and Technology (NTNU), \\ 7491 Trondheim - Norway \\ e-mail: loic.barre@ifp.fr - jacques.jestin@cea.fr - anne.morisset@ifp.fr - thierry.palermo@ifp.fr - sebastien.simon@chemeng.ntnu.no
}

\begin{abstract}
Résumé - Relation entre nanostructure des agrégats d'asphaltène et les propriétés macroscopiques de leur solution - Certaines propriétés particulières de bruts pétroliers sont attribuées à leur fraction la plus dense, lourde et polaire : les asphaltènes. La compréhension de l'origine de ces propriétés repose sur une description structurale fine de ces fractions. Nous présentons une caractérisation des solutions d'asphaltène, à l'échelle du nanomètre, basée sur de nouvelles ou récentes expériences de diffusion de rayonnement (rayons $\mathrm{X}$ ou neutrons) à partir desquelles des paramètres structuraux des agrégats d'asphaltène $\left(R_{g}, M_{w}\right.$ et $\left.A_{2}\right)$ sont déduits. La dépendance de ces paramètres est compatible avec un modèle d'agrégats de type fractal de masse. Ce modèle rend compte de toute la variété de propriétés macroscopiques de ces solutions. En particulier, la prise en compte de l'aspect solvaté des agrégats permet de prédire la viscosité de solutions d'asphaltène en fonction de leur concentration. D'autre part, les asphaltènes s'adsorbent aux interfaces liquide-liquide et liquide-solide et forment des monocouches dont l'épaisseur est du même ordre de grandeur que la taille des agrégats de la solution. L'analyse des isothermes d'adsorption, les expériences de réflectivité des neutrons ainsi que les expériences de diffusion des neutrons en conditions d'extinction de contraste, mettent en évidence une densification des agrégats d'asphaltène dans la couche adsorbée relativement à leur état solvaté dans le volume. Enfin, la résultante des interactions attractives/répulsives entre agrégats peut être reliée à la stabilité relative d'émulsions d'eau dans l'huile et à la stabilité d'effluents d'hydroconversion. Cette approche de mise en relation entre structure et propriété permet d'asseoir et de justifier le modèle de fractal de masse qui est discuté.
\end{abstract}

\begin{abstract}
Relation between Nanoscale Structure of Asphaltene Aggregates and their Macroscopic Solution Properties - Some peculiar macroscopic properties of crude oils are ascribed to their densest, heaviest and most polar fraction: the asphaltenes. The comprehension of the origin of these properties relies on a fine structural description of these fractions. We present a nanometre length scale characterisation of asphaltene solutions based on new or recent scattering experiments $(X$-rays and neutrons) from which structural parameters of asphaltene aggregates $\left(M_{w}, R_{g}\right.$ and $\left.A_{2}\right)$ are extracted. The mutual dependence of these parameters is consistent with a mass fractal model. This single model accounts for very different macroscopic solution properties. Solvent trapping in fractal aggregates enables asphaltene solution viscosity to be fully predicted as a function of asphaltene concentration. Asphaltene adsorbs at liquid-liquid and liquid-solid interfaces as a monolayer whose thickness is of the
\end{abstract}


same order of magnitude as the characteristic size of the asphaltene aggregate in bulk. Adsorption isotherm analysis, neutron reflectivity experiments and contrast-matching SANS allow one to highlight a densification of the asphaltene aggregates in the adsorbed layer in comparison with the solvated aggregates in bulk. Finally, the repulsivelattractive interaction balance between aggregates can be related to the relative stability of water in oil emulsions and to the stability of hydro-conversion effluents. This structure/property approach allows one to assess the mass fractal model which is discussed.

\section{INTRODUCTION}

The growing demand for fuels and the supply of heavier crude oils require the development of new processes to enhance the production, the transport and the refining of these products. However, these developments are made difficult by the intrinsic properties of heavy oils.

In the upstream industry, heavy oils are known [1] to be responsible for high viscosities of heavy crude oils [2], to be involved in wettability alteration of mineral surfaces in reservoir formation and in formation and high stability of water in oil emulsions [3, 4]. Heavy oils are also at the origin of the plugging problem via deposition in porous media during production [5] and are thought to affect stability of gas hydrate particles in oil production pipelines, thus preventing the formation of solid plugs which would result in the blockage of the lines [6].

In the downstream sector, handling heavy cuts represents a problem in respect to their high viscosity and their tendency to metal surface fouling. Moreover, they constitute the most resistant fraction to hydroconversion because diffusion of some species in the catalyst porous network is thought to be limited. Finally, any hydroconversion process is limited, at high conversion levels, by insoluble solid formation.

The shared characteristic of these products is their asphaltene content, which confers on them peculiar properties. Asphaltenes are defined by their insolubility in normal alkanes. Chemical analysis indicates that this fraction concentrates the most aromatic and polar molecules [7]. The determination of asphaltene molecular weight has been a subject of intense debate and it is now generally agreed that values are 500 to $1000 \mathrm{~g} \cdot \mathrm{mol}^{-1}[8,10]$. Furthermore, most of the molecules have a central aromatic part with peripheral aliphatic or naphthenic moieties $[8,10]$.

Their behaviour in solution is quite complex. At very low concentration and even in "good solvents" such as toluene, above a concentration known as the critical nano-aggregate concentration (CNAC) $c \approx 100 \mathrm{mg} / 1$, these molecules selfassociate to form nano-aggregates $[11,12]$. At higher concentrations, these nano-aggregates form larger structures whose size and mass depend on thermodynamic conditions (pressure and temperature), on the nature of the solvent in which they are suspended $[13,14]$ or on the presence of resins [15]. These structures will be referred to as aggregates in this article.
Several methods can be used in order to characterise the asphaltene aggregate structure and their interactions. Among them, scattering techniques are well suited for concentrated and opaque systems. The first part of this paper is devoted to their presentation, with particular emphasis on the length scale and contrast considerations. A simple and robust method of data analysis, which allows one to extract meaningful structure parameters, will be discussed.

We present recent or new results aiming to understand better or to predict macroscopic properties of asphaltenic fluids from nanoscale aggregate description and using parameters inferred from scattering measurements. This structure/function approach is illustrated by several examples chosen in different areas of the petroleum industry. A structural model relying on these results will be discussed.

\section{STRUCTURAL INFORMATION FROM SCATTERING MEASUREMENTS}

Several different methods have been applied to determine the molecular mass of asphaltenes and/or their different aggregation states: vapour pressure osmometry [16], small-angle neutron and X-ray scattering (SANS and SAXS, see below), mass spectrometry [8-16], fluorescence depolarisation measurements $[9,10]$, and NMR $[17,18]$. Mass results are different in regard to the concentration range and the measurement technique. The mass variation observed as a function of the concentration has been assigned to aggregation, whereas the specificity of each technique (sensitivity to hydrogen content, to different moments of size distribution, etc.) could account for deviations at a given concentration. Among these methods, SANS and SAXS can operate at asphaltene concentration up to $20 \mathrm{wt} . \%$ [25] and thus constitute well-adapted techniques to study asphaltene aggregates in relation to properties of concentrated asphaltenic fluids. In the low concentration range, measurements down to $0.5 \mathrm{wt} . \%$ for SAXS [25] or to $0.3 \mathrm{wt} . \%$ for SANS [4] have been reported.

They have been widely used to probe the nanoscale organisation of heavy crude oils or asphaltene solutions [13, 19-22]. Recently, an interpretation of scattering spectra in terms of local ephemeral fluctuation has been proposed [23, 24]. Apart from that, most of the studies agreed on a colloidal view of asphaltene aggregates with characteristic sizes of 3 to $10 \mathrm{~nm}$, depending on the asphaltene and solvent nature, and on thermodynamic conditions. Accordingly, the measured 
masses vary from 50 to $200 \mathrm{kDa}$. These studies pointed out the large size polydispersity [20] of these aggregates, but there are less studies concerning interactions between aggregates [25].

The asphaltene aggregate form factors have been inferred from these measurements but the variety (spheres [25], oblate or prolate [22] cylinders, mass fractal aggregates [20]) of solutions indicates that aggregate geometry is poorly determined. Recently, we proposed a constrained method based on additional structural viscosity data [25] which shows that both scattering and viscosity data are consistent with a mass fractal aggregate description.

The following part describes a methodology to analyse scattering spectra (neutrons or X-rays) without assumption on particle shape and taking into account particle interactions. The parameters deduced from scattering measurements are discussed.

\subsection{Neutron and/or X-ray Scattering}

Neutron and X-ray scattering experiments measure the scattered intensity $I(q)$ which probes the correlations between asphaltene-rich regions on a scale of the order of $q^{-1}$. The scattering vector $q$, defined as $4 \Pi \sin \theta / \lambda$ where $2 \theta$ is the deviation angle and $\lambda$ the incident wavelength, acts as a reverse length scale. While SAXS and SANS offer a typical $q$ range that is very similar (see Fig. I), they differ above all in the beam/matter interaction nature: X-rays interact with electrons, whereas neutrons interact with nuclei. The relevant characteristics of the probed medium are, respectively, the electronic density $\left(d_{e}\right)$ or the scattering length density $\operatorname{SLD}\left(\rho_{\mathrm{N}}\right)$ fluctuations which give rise to the scattering. For a two-component medium labelled by subscripts 1 and 2 , the contrast term $\Delta \rho^{2}$, to which scattered intensity is proportional, is simply $I_{e}\left(d_{e 1}-d_{e 2}\right)^{2}$ for X-rays and $\left(\rho_{\mathrm{N} 1}-\rho_{\mathrm{N} 2}\right)^{2}$ for neutrons, where $I_{e}$ is the scattered intensity by a single electron $\left(7.9 \times 10^{-26} \mathrm{~cm}^{2}\right)$.

The $d_{e}$ and $\rho_{\mathrm{N}}$ values for each component can be estimated assuming that the chemical composition and densities are known [14]. Table 1 summarises the contrast terms for X-rays and neutrons for heptane-insoluble Safanyia asphaltenes in hydrogenated or deuterated forms of toluene. The contrast term is enhanced using neutron and deuterated solvents but is still usable if $\mathrm{X}$-rays and hydrogenated toluene are considered. Figure 1 is a comparison of a few percent of asphaltene either in toluene and measured by SAXS, or in deuterated toluene and measured by SANS. These measurements were made for asphaltene precipitated from the same crude oil by an excess of pentane (iC5), heptane (iC7) or octane (iC8). The scattered intensities are normalised by the volume fraction of asphaltene $(\phi)$ and contrast terms which vary according to their composition and densities [4].

The X-ray and neutron curves are superimposed at small $q$ values but differ slightly at large $q$. These differences can be
TABLE 1

Comparison of X-ray and neutron contrast terms for Safanyia asphaltene in hydrogenated or deuterated toluene

\begin{tabular}{c|c|c}
\hline & $I_{e}\left(d_{e 1}-d_{e 2}\right)^{2}\left(\AA^{-4}\right)$ & $\left(\rho_{1}-\rho_{2}\right)^{2}\left(\AA^{-4}\right)$ \\
\hline Asphaltene $/ \mathrm{C}_{7} \mathrm{H}_{8}$ & $8.15 \times 10^{-12}$ & \\
\hline Asphaltene $/ \mathrm{C}_{7} \mathrm{D}_{8}$ & & $18.1 \times 10^{-12}$ \\
\hline
\end{tabular}

explained on a basis of particle heterogeneity. Indeed, the Yen micelle [27] or the recently proposed nano-aggregate [28] are based on the formation of aggregates by $\pi-\pi$ interactions of fused aromatic rings which, as a consequence, have peripheral aliphatic or naphthenic moieties. For such a particle, the electronic density is larger in the central

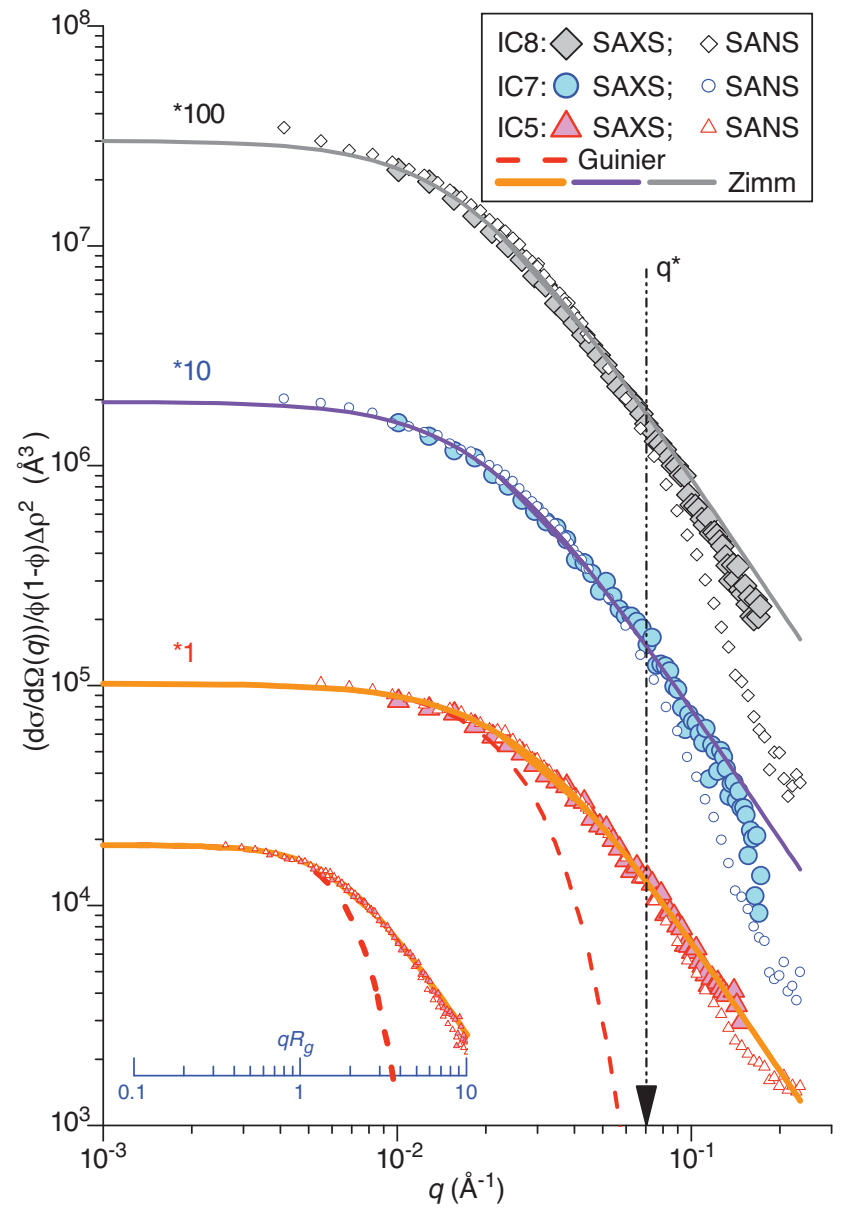

Figure 1

Comparison of SAXS and SANS spectra: variations in $I(q) / \phi \Delta \rho^{2}$ as a function of the wave scattering vector $q$ for solutions of different asphaltenes (see text) in toluene. The dotted and full lines represent, respectively, the Guinier (Eq. 2) and the $\operatorname{Zimm}(E q .3)$ approximation in the small $q$ domain. 
(aromatic) part than in the peripheral one (aliphatic), whereas the neutron scattering length density is inversely distributed. These heterogeneities on a small scale (a few Angstroms) may result in a contrast-dependent signal at large $q$ values, whereas their average on a larger length scale (small $q$ values) may result in a contrast-independent signal. For the asphaltenes considered in Figure 1, the crossover between these two regimes, noted $q^{*}$, is close to $7 \times 10^{-2} \AA^{-1}$, which corresponds in the direct space to size heterogeneity smaller than $15 \AA\left(1 / q^{*}\right)$.

\subsection{Form Factor and Approximations}

The general expression for a two-phase system of particles, at volume fraction $\phi$, in a solvent is:

$$
l(q)=\phi(1-\phi) \Delta \rho^{2} F(q) \mathrm{S}(q)
$$

where $S(q)$ is the structure factor which takes into account interactions between particles and $F(q)$ is the form factor normalised to the "scattering volume" v (i.e. $F(0)=v$ ). At small concentration, interactions between particles can be neglected $(S(q) \rightarrow 1)$. As seen previously from neutron and $\mathrm{X}$-rays comparisons, the form factor may be rather complex due to small scale heterogeneity, and we preferably use approximations of the form factor at small $q$ values. Two of them are available and plotted in Figure 1; respectively, the Guinier and the Zimm approximation:

$$
F(q)=F(0) \exp \left(-\frac{q^{2} R_{g}^{2}}{3}\right) \text { for } q R_{g}<1
$$

(Guinier approximation)

$$
\frac{1}{F(q)}=\frac{1}{F(0)}\left(1+\frac{q^{2} R_{g}^{2}}{3}\right) \text { for } q R_{g}<1
$$

(Zimm approximation)

If these two expressions are equivalent for $q R_{g}<1$ (see the inset in Fig. 1), the Guinier approximation fails to describe the data for $q R_{g}>1$, whereas the Zimm approximation is still valid for both SAXS and SANS measurements up to $q R_{g}=3$. This behaviour, already observed in the polymer solution area [29], extends the validity domain of the Zimm approximation for asphaltene solutions and enables a robust and model-independent method to analyse the scattering curves: the $1 / I(q)$ versus $q^{2}$ plot gives directly the radius of gyration $R_{g}$ of the particles and the extrapolation of measurement at $q=0$ enables one to obtain $v$ knowing the contrast term $\Delta \rho^{2}$. The "mass" $M_{w}$ of aggregates is deduced from $v$ and asphaltene density $d$ by the usual expression:

$$
M_{w}=N_{a} d v
$$

where $N_{a}$ is the Avogadro number. Combining (1), (3) and (4) and considering concentration $c$ in grams per unit volume, one obtains:

$$
\frac{\Delta \rho^{2}}{d^{2} N_{a}} \frac{c}{I(q)}=K \frac{c}{I(q)}=\frac{1}{M_{w}}\left(1+\frac{q^{2} R_{g}^{2}}{3}\right) \text { for } q R_{g}<3
$$

This expression constitutes a robust and practical way to estimate sizes and masses as far as dilute asphaltene solutions are considered.

\subsection{Interactions}

For more concentrated systems, the inter-particle interactions have to be taken into account and the previous equation is modified through a virial expansion to give:

$$
\begin{gathered}
\frac{I_{e} \Delta \rho^{2}}{d^{2} N_{a}} \frac{c}{I(q)}=\frac{1}{M_{w}}\left(\left(1+\frac{q^{2} R_{g}^{2}}{3}\right)+2 A_{2} M_{w} c+\ldots\right) \\
A_{2} M_{w} c<0.25
\end{gathered}
$$

This equation can be simplified in the Guinier region to yield:

$$
\begin{gathered}
\frac{I_{e}\left(\Delta \rho^{2}\right)}{N_{a} d^{2}} \frac{c}{I(0)}=\frac{1}{M_{w}}\left(1+2 A_{2} M_{w} c\right)=\frac{1}{M_{a p p}(c)} \\
q R_{g}<1 ; A_{2} M_{w} c<0.25
\end{gathered}
$$

where $A_{2}$ is the second virial coefficient. Positive values of $A_{2}$, related to repulsive interaction between objects, leads to a lowering of the $I(q) / \phi$ ratio at small $q$ values when the concentration increases. This relation holds up to concentrations where interactions are described by pair interactions. The left-hand part of Equation (7) can be used to define an "apparent" mass $M_{a p p}$ which tends toward the real one at low concentration.

\subsection{Mass Fractal Aggregates}

Asphaltene particles have been frequently described as mass fractal aggregates of dimension $D_{f}[2,13,20,22,30]$. In this case, on a much smaller scale than the typical size of aggregates, a simple expression of the scattering cross-section is expected:

$$
I(q) \propto q^{-D_{f}} \quad q R_{g}>1
$$

Furthermore, a power law relation between the measured aggregate parameters, namely, radius of gyration, second virial coefficient and molar mass, should be observed for these aggregates [29]:

$$
\begin{gathered}
R_{g}=k_{2} M_{w}^{1 / D_{f}} \\
A_{2}=k_{3} M_{w}^{-\left(\frac{3}{D_{f}}-2\right)}
\end{gathered}
$$




\subsection{Polydispersity Effects}

The size polydispersity has a notable influence on scattering properties of a suspension. In the dilute regime $(S(q) \rightarrow 1)$, expression (1) can be extended:

$$
I(q)=\Delta \rho^{2} \sum_{k} n_{k} v_{k} F_{k}(q)
$$

where $n_{k}$ and $v_{k}$ are, respectively, the number of particles per unit volume and the volume of each $k$ entity. For simple form factors such as homogeneous spheres, size distribution could be extracted providing the repartition function has a simple and analytical form such as Gaussian, Log-normal or Schulz distributions.

In practice, for polydisperse systems such as asphaltene solutions [20, 31], Guinier or Zimm approximations give the weight-average $M_{w}$ molecular weight of aggregates and the $z$-average radius of gyration $R_{g z}$ defined by the following expressions:

$$
M_{w}^{2}=\frac{\sum_{k} n_{k} M_{k}^{2}}{\sum_{k} n_{k}}
$$

and

$$
R_{g z}^{2}=\frac{\sum_{k} n_{k} v_{k}^{2} R_{k}^{2}}{\sum_{k} n_{k} v_{k}^{2}}
$$

with $M_{k}, R_{k}$ : the molar mass and radius of gyration of the $k$ fraction, respectively.

Asphaltene solution polydispersity has been evaluated using ultracentrifugation [20, 25] or nano-filtration [32] fractionation followed by a SAXS analysis of the fractions. It has been found that size distribution is large and obeys a power law.

\section{MACROSCOPIC PROPERTY PREDICTIONS FROM MICROSCOPIC STRUCTURAL DETERMINATION}

Macroscopic properties of colloidal solutions are generally related to size, mass and interactions of their constituting objects, and a structural solution description on a pertinent length scale enables one to understand better and predict the properties.

It has been shown that asphaltenic fluids belong to this more general class of colloidal solutions. Unfortunately, structural description of these complex fluids remains difficult because of the large number of their constituents, their viscosities, their blackness, etc. Our approach is a simplification by extraction of asphaltene from these fluids and dissolution in simple solvents in order to access the structural parameters, as described in the previous section. If the solution behaviour captures basic properties of parent asphaltenic fluid, knowledge of structural solution parameters is thought to be relevant to predict macroscopic properties of the real fluids. This structure/properties relation approach in the domain of asphaltenic fluids is illustrated by a few recent examples chosen in different areas of the petroleum industry.

\subsection{Viscosity}

Viscosity prediction is crucial for upstream or downstream process development. It has been shown that the asphaltene fraction is mainly responsible for large viscosity of heavy crude oils or residues $[2,33]$ and the colloidal particle suspension approach has been used in order to explain the viscosity dependence on asphaltene concentration [34, 35]. In this approach, the relative viscosities $\eta_{\mathrm{r}}$ of asphaltene suspensions, defined as the ratio of the solution viscosity $\eta$ to that of pure solvent $\eta_{0}$, are measured as a function of the volume fraction $\phi$ of the solute. For a low concentration regime, the Huggins expression is used:

$$
\frac{1}{\phi}\left(\frac{\eta}{\eta_{0}}-1\right)=[\eta]+k[\eta]^{2} \phi+\ldots
$$

where $[\eta]$ is the so-called intrinsic viscosity and $k$ the Huggins constant.

For homogeneous spheres, $[\eta]$ is the well-known Einstein [36] coefficient and its numerical value is $5 / 2$. If the suspended particles are not spheres, different values of $[\eta]$ have to be considered. In particular, for mass fractal aggregates, intrinsic viscosity depends on mass $M$ (or size $R$ ) and fractal dimension $D_{f}$ :

$$
[\eta]=k_{1} M^{\frac{3}{D_{f}}-1}
$$

For solvated particles or aggregates, the hydrodynamic "swollen" volume, often called "effective" volume $v_{\text {eff }}$, is different from the "dry" volume $v$ as determined by scattering experiments. The ratio of these two volumes is the so-called solvation constant $k_{s}$. In the same way, the effective $\phi_{\text {eff }}$ and nominal $\phi$ volume fraction can be defined. So, for a dilute system of spherical solvated aggregates, Equation (15) can be reformulated:

$$
\begin{gathered}
\eta_{r}=\frac{\eta}{\eta_{0}}=1+\frac{5}{2} \phi_{\text {eff }}+\ldots=1+\frac{5}{2} k_{s} \phi+\ldots=1+[\eta] \phi+\ldots \\
\text { and } \phi_{\text {eff }}=\frac{[\eta]}{2.5} \phi
\end{gathered}
$$

These latter equations supply practical ways to convert nominal volume fractions into effective ones.

The Huggins coefficient $k$ is a measurement of both hydrodynamic and "thermodynamic" interactions between particles. For mono-dispersed hard sphere systems, Batchelor [37] found a value of 1.2. This second-order coefficient takes suspension viscosity behaviour into account up to $\phi=0.15$. 
For more concentrated systems, semi-empirical models have been developed. They predict the viscosity divergence when the particle volume fraction approaches the dense random packing limit fraction $\phi_{m}$. The Quemada equation [38], based on dissipated energy minimisation by viscous effects, is frequently used because of its simplicity:

$$
\eta_{r}=\left(1-\frac{\phi}{\phi_{m}}\right)^{-2}
$$

Parameters such as $k$ and $\phi_{m}$ depend closely on particle size polydispersity.

While this colloidal particle suspension approach succeeded in asphaltene solution viscosity description [39], only a few studies have focused on linkage between structural parameters and the corresponding viscosity. This coupling approach constrains the rheological models and allows one to assess a more realistic structure to these asphaltene aggregates. We present in the following the main results [25] obtained by a combination of scattering experiments, from which the structural parameters $M_{w}, R_{g}, A_{2}$ and $D_{f}$ have been determined, and viscosimetric measurements from which $[\eta], k$ and $\phi_{m}$ have been inferred. These determinations were conducted on asphaltene fractions obtained by ultracentrifugation. This fractionation technique allows enough material of reduced polydispersity and varied mass to be obtained to study concentration dependence of viscosity and X-ray scattering in the range 1-15 wt $\%$.

All the SAXS diagrams show a Guinier regime from which $M_{w}, R_{g}$ and $A_{2}$ are determined using the Zimm approximation as described in Section 1. At large $q$ values, all the intensities, when normalised with respect to concentration, merge on a master curve and follow a $q^{-2.1}$ decay. For each fraction, the relative viscosities follow a similar exponential increase which can be fitted with Equations (15) and (17) at low and intermediate concentration and (18) at high concentration.

The values of $M_{w}, R_{g}, A_{2}$ and $[\eta]$ and their dependence are presented in Figure 2. Despite the small number and limited range of data, a regression analysis yields the following relationships:

$$
\begin{gathered}
R_{g}=0.43 M_{w}^{0.45} \\
{[\eta]=0.049 M_{w}^{0.41}} \\
A_{2}=0.0129 M_{w}^{-0.55}
\end{gathered}
$$

As previously explained in Section 1, all these power law type relations could be related to mass fractal organisation of the aggregates. Comparison of relations $(19,20,21)$ with Equations $(9,16,10)$ leads, respectively, to the fractal dimensions $D_{f R g}=2.2, D_{f[\eta]}=2.1$ and $D_{f A 2}=2.1$. The closeness of these different independent determinations of fractal dimension, $D_{f R g} \approx D_{f[\eta]} \approx D_{f A 2}$, close to the 2.1 exponent determined from $q$ dependence of intensities at

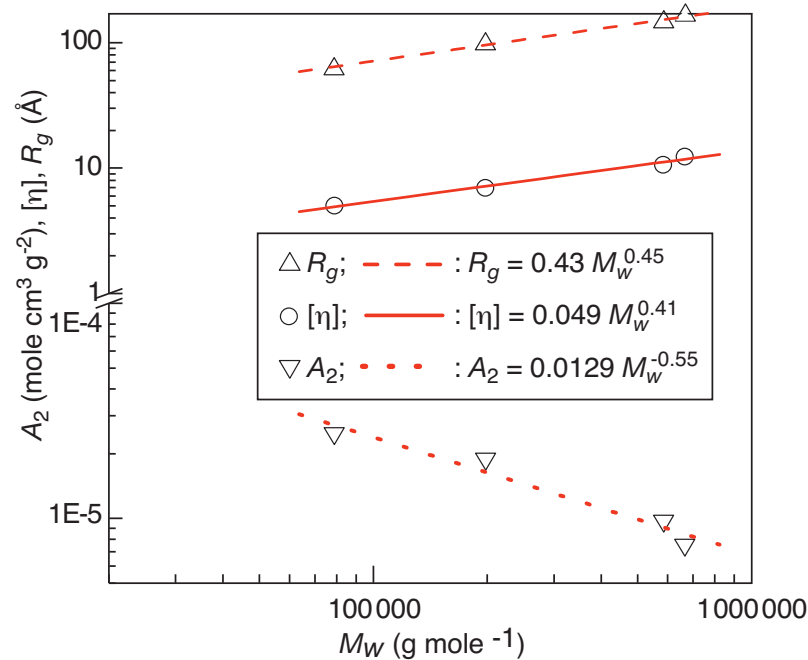

Figure 2

Relations between the intrinsic viscosity $[\eta]$, the radius of gyration $R_{g}$, the second virial coefficient $A_{2}$, and the mass $M_{w}$ of asphaltene aggregates in toluene at $25^{\circ} \mathrm{C}$ (from [25]).

large $q$ values, is a very strong indication of the fractal nature of asphaltene aggregates. It can be concluded that asphaltene solutions in toluene are described as a polydispersion of solvated aggregates characterised by a fractal dimension close to 2.1. Moreover, the solution viscosities at low concentrations are controlled by the size, mass and fractal structure of the aggregates.

The consequence of the swollen nature of aggregates has to be taken into account for both viscosity and scattering properties. The pertinent parameter which takes into account the concentration of aggregates entrapping solvent is the effective volume fraction $\phi_{\text {eff }}$. If relative viscosities are plotted versus this parameter using Equation (17) to convert nominal into effective volume fractions, it is shown that any fraction at any concentration merges on the single curve of hard spheres represented by Equations 15 and 18. Similarly, if the so-called "reduced osmotic modulus" $[29,4]$ is plotted as a function of a reduced concentration $X=A_{2} M_{w} c$, all the scattering data merge on a master curve which is well represented by a hard sphere model. These two observations are in favour of hard sphere interactions, and consequently no overlapping between aggregates.

\subsection{Adsorption of Asphaltene Aggregates at the Solid/Liquid Interface}

Asphaltenes are known to adsorb from crude oils onto solid surfaces. This phenomenon is at the origin of macroscopic property variations encountered in the petroleum industry such as wettability alteration of mineral surfaces in reservoir 
formation $[40,41]$ or stabilisation of gas hydrate particles in oil production pipelines, thus preventing the formation of solid plugs which would result in the blockage of the lines [6].

The numerous studies that have been conducted in the past have shown that asphaltene, when dissolved in good solvents, adsorbs on polar solids until the surface is saturated. The corresponding isotherms are usually Langmuir type and the typical plateau adsorbed amount, at a few $\mathrm{g} / \mathrm{l}$ equilibrium concentrations, is close to $2-4 \mathrm{mg} / \mathrm{m}^{2}$.

While the isotherm approach has enabled the determination of the influence of parameters on the asphaltene adsorbed amount [42-44], less is known about the structure of the asphaltene layer. We present in the following the main results of recent studies $[45,46]$ showing the relation between the solution and interfacial properties of asphaltene aggregates.

In the first study [46], we considered asphaltenes of different origin, in different solvents and including different amounts of resins. Asphaltene's adsorption properties were explored by measuring adsorption isotherms onto silica particles. The affinity constant and plateau adsorbed amounts $\Gamma_{\max }$ are inferred from these measurements. Volume properties, radius of gyration $R_{g}$ and mass $M_{w}$ of the aggregates are deduced from SAXS measurements using the Zimm formalism as explained in Section 1. Additional characterisation including specific gravity measurement was also conducted.

The volume properties of these asphaltene solutions were first explored: the radius mass dependence was fitted by the relation:

$$
R_{g}=0.41 M_{w}^{0.45}
$$

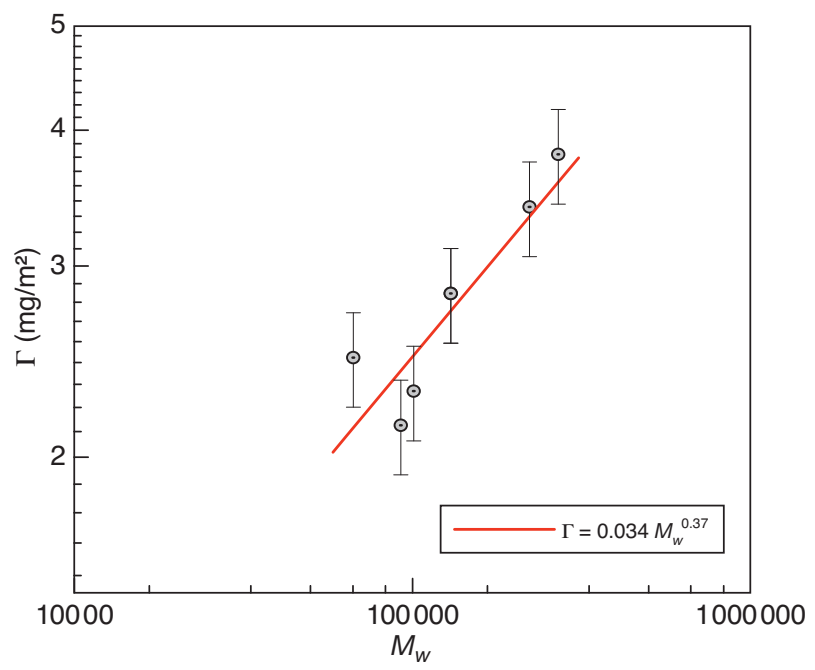

Figure 3

Variations in the plateau adsorbed amount $\Gamma_{\max }$ as a function of mass of asphaltene aggregates $M_{w}$ in different solvents and in the presence of different resin contents (from [46]).
Identification of relation (22) with Equation (9) leads to a value of fractal dimension $D_{f}=2.2$, very close to that determined from the viscosity study; the pre-factor $(0.41)$ also has a numerical value comparable (0.43) with that obtained for fractions obtained by centrifugation (see Sect. 2.1). This observation strengthens the fractal behaviour developed in the previous section and gives it a more general character. Figure 3 presents, for one asphaltene of a given origin in different solvents and in the presence of different resin contents, the dependence of excess amount at surface saturation $\Gamma_{\max }$ as a function of aggregate mass $M_{w}$ in solution. There is a clear correlation between these two quantities, suggesting aggregate adsorption, and consequently the presence of a monolayer of aggregates at the solid/liquid interface. Moreover, a power law can be extracted from this dependence:

$$
\Gamma_{\max }=0.034 M_{w}^{0.37}
$$

A model of aggregate adsorption based on geometrical considerations was developed to account for experimental observations. If all the surface $S$ is paved with $n$ aggregates of mass $M_{w}$ and size $R_{s}$, the plateau absorbed amount is:

$$
\Gamma_{p l}=\frac{m_{\text {adsorbed }}}{S}=\frac{n M_{w}}{n N_{a} \pi R_{s}^{2}}
$$

Note that in this approach, $R_{s}$ is allowed to be different from $R_{g}$ if some structural modification occurs during adsorption. In this case, $R_{s}$ is related to the mass $M_{w}$ and to the fractal dimension $D_{s}$ of aggregates in the adsorbed state by:

$$
R_{s}=\frac{1}{p} M_{w}^{\frac{1}{D_{s}}}
$$

where $p$ is a prefactor. The combination of Equations (24) and (25) leads to an expression whose form is similar to (23):

$$
\Gamma_{p l}=\frac{p^{2}}{N_{a} \pi} M_{w}^{\left(1-\frac{2}{D_{s}}\right)}
$$

Identification of the exponent value in relation (23) and Equation (26) lead to a numerical value of $D_{s}$ close to 3 , which means that aggregates lose their fractal character in the adsorbed state. Asphaltene volume fractions in the adsorbed layer were estimated with the help of additional mass density data: it has been shown that the volume fraction of asphaltene changes from a few percent in volume aggregate to roughly 40 percent in the adsorbed layer.

In the second study [45], we considered asphaltene solution in contact with silicon wafers. This liquid-solid interface was probed using neutron reflectivity from which Scattering Length Density (SLD) profiles were obtained. Figure 4a represents reflectivity $R$ as a function of scattering vector $q$ for asphaltenes extracted from crude oils using different alkanes (iC5, iC7 and iC8) and dissolved in deuterated xylene at $0.3 \mathrm{wt} . \%$. The $R q^{4}$ representation enables differences from Fresnel reflectivity to be enhanced 

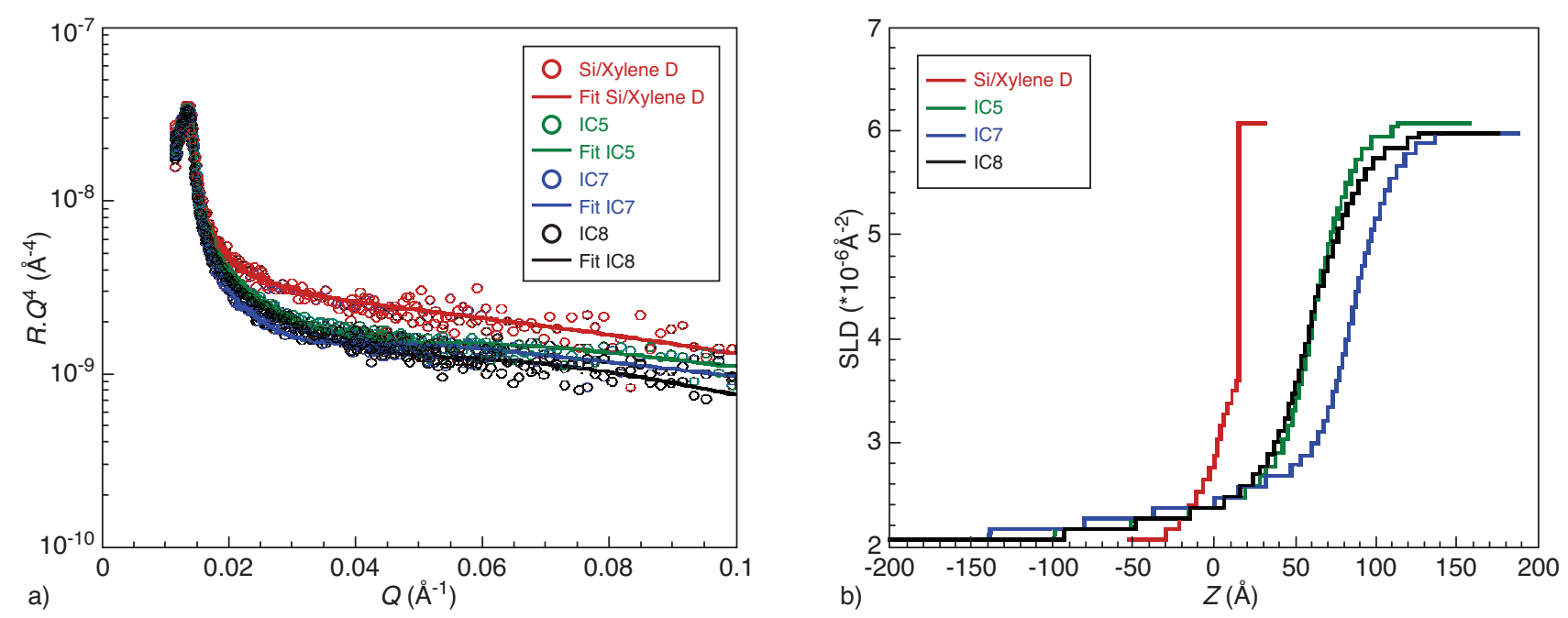

Figure 4

a) $R_{q}{ }^{4} \log$-log representation of asphaltene absorbed on hydrophilic surfaces without asphaltene (red), IC5 (green), IC7 (blue), IC8 (black). The solid lines are the profiles calculated with a monolayer model, b) the resulting Scattering Length Density (SLD) profiles were deduced from the best fitting procedure.

[45]: in the present case, addition of asphaltene to the solution leads to a slight decrease in reflectivity at large $q$ values, which is the signature of asphaltene adsorption. These data were analysed successfully using a simple model based on a rough monolayer of constant scattering length density. The corresponding fitted curves are presented as a full line in the same figure. The values of regressed parameters (Fig. 4b) indicate a thickness of the adsorbed layer of a few nanometres, in accordance with the radii of gyration of aggregates in the volume and a roughness of this layer consistent with the large size polydispersity of aggregates in toluene [20,25]. Moreover, the mean scattering length density of the adsorbed layer is found to be consistent with the densification observed in the previous study [46].

\subsection{Stabilisation of Petroleum Emulsions by Asphaltene Aggregates}

Heavy crude oils or heavy cuts, when mixed with water, are known to produce a very stable water in oil emulsion. The origin of this long-term stability has been assigned to elastic and viscoelastic properties of the interfacial film as studied by interfacial rheology [47-49]. Surface species have been found to be composed of high-molecular-weight (400-700 Da) molecules containing mostly carboxylic and thiophenic groups [50]. It has also been shown that the asphaltene fraction, when solubilised in aromatic solvents, can form a stable emulsion [51]. Finally, Kilpatrick's group [15, 52, 53] has demonstrated that emulsion stability depends on the aggregation state of asphaltenes, but very little is known on the mechanism on the length scale of aggregates. We present in the following the main results of a recent study [4] aiming at establishing relations between the structure of asphaltene aggregates in the volume, surface film characteristics and macroscopic stability of emulsions.

Emulsions were prepared by mixing controlled $\mathrm{pH}$ water with different model oils (asphaltene in xylene). The aggregate sizes of model oils were varied by using different precipitants (pentane, heptane or octane) during asphaltenemaltene separation (samples iC5, iC7 and iC8, respectively). Centrifugation, in controlled conditions, and subsequent weighting of free separated water were used to classify emulsion stabilities according to their relative water quantities recovered. The powerful SANS technique in contrast-matching conditions was used in order to get the signal scattered only by the interfacial films from which its thickness $H$ and the mean amount of solvent were deduced. The characteristics of the corresponding volume aggregates ( $M_{w}, R_{g}$ and $A_{2}$ ) were inferred from SAXS measurements as described in Section 1. It was found that film thickness values (113 to $149 \AA$ ) are directly related to the radii of gyrations $R_{g}$ of asphaltene aggregates in the bulk phase (66 to $106 \AA$ ). This is a strong indication that the interfacial film is made up of a monolayer of aggregates. This idea is reinforced by the specific adsorption of the larger aggregates illustrated by the decrease in the mean bulk aggregate sizes after the emulsification process. Moreover, it has been proposed that stability of these different emulsions is related 


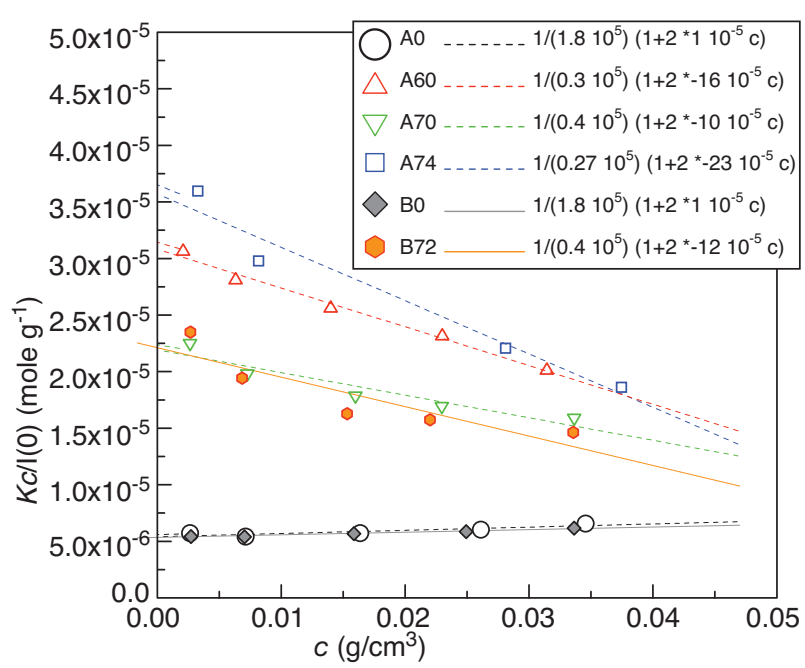

Figure 5

Reverse apparent mass of aggregates as a function of concentration in THF (see Eq. 7) for asphaltenes extracted both from feed and effluent.

to interaction of asphaltene aggregates in the interfacial film. The attractive/repulsive balance between the aggregates in the film, thought to be reflected by the different $A_{2}$ values in the volume, has a direct incidence on the quantity of water recovered after centrifugation: the stronger the attraction between aggregates in the film, the more stable the emulsion.

\subsection{Colloidal Stability}

The precipitation of asphaltene aggregates is a limiting mechanism for different processes developed in the petroleum industry. This precipitation phenomenon, close to flocculation onset conditions, leads to time-dependent aggregation in the volume $[54,55]$. It has been shown recently $[45,56]$ that these conditions favour deposition at the solid/liquid interface by a multilayer growth process. In the refining domain, flocculation is known to limit the conversion of heavy cuts into lighter ones in the H-Oil process [57]. As described in part 1, small-angle scattering has the ability to gain insight into the origin of colloidal stability via $A_{2}$ measurements.

Asphaltene fractions, defined here as part of the residue precipitated in 40 volumes of heptane at reflux temperature for 15 minutes, were isolated both from feedstocks and effluents obtained in the H-Oil process at different conversion levels. The asphaltenes were then dissolved in tetrahydrofuran (THF) at concentration up to $0.04 \mathrm{~g} / \mathrm{ml}$ and were allowed to stand overnight. Observation of these solutions using an optical microscope allowed one to verify the sub-micronic dispersion state of asphaltene.
TABLE 2

Chemical composition and densities of asphaltene extracted from feeds or effluents at different conversion levels

\begin{tabular}{c|c|c|c|c|c|c|c|c}
\hline Feed & $\begin{array}{c}\% \text { Conv } \\
540+\end{array}$ & $d\left(\mathrm{~g} \mathrm{~cm}^{-3}\right)$ & $\% \mathrm{C}$ & $\% \mathrm{H}$ & $\% \mathrm{~N}$ & $\% \mathrm{O}$ & $\% \mathrm{~S}$ & $\mathrm{H} / \mathrm{C}$ \\
\hline $\mathrm{A}$ & 0 & 1.23 & 85.9 & 7.36 & 1.33 & 1.75 & 3.30 & 1.02 \\
\hline & 60 & 1.32 & 90.9 & 5.96 & 1.23 & 1.34 & 0.55 & 0.78 \\
\hline & 70 & 1.30 & 91 & 5.75 & 1.34 & 1.37 & 0.76 & 0.76 \\
\hline & 74 & 1.34 & 90.9 & 5.61 & 1.42 & 1.16 & 0.86 & 0.74 \\
\hline $\mathrm{B}$ & 0 & 1.21 & 85.7 & 7.65 & 1.39 & 1.44 & 3.44 & 1.07 \\
\hline & 72 & 1.36 & 89.9 & 6.05 & 1.62 & 1.04 & 1.54 & 0.81 \\
\hline
\end{tabular}

The elemental analysis of these asphaltene fractions, as reported in Table 2, shows large variations in the $\mathrm{H} / \mathrm{C}$ ratios, from 1.07 for feeds up to 0.74 for effluents obtained in severe hydroconversion conditions.

SAXS data were collected using homemade equipment. A copper rotating anode generator (Rigaku Micromax 007), operating at $0.8 \mathrm{~kW}$, provides an $\mathrm{X}$-ray beam which is reflected on a Xenocs parabolic multilayer mirror. The reflected monochromatic beam $(\lambda=1.54 \AA)$ is collimated by two pairs of crossed slits whose parasitic scattering is removed by another pair of crossed slits located just before the sample. The asphaltene solutions were loaded in 2-mm glass capillaries. A Rigaku 2D position-sensitive detector, located $1.2 \mathrm{~m}$ from the sample, collected the scattering intensity. The range of scattering angles $2 \theta$ enables a range of wave scattering vector $q$ of $210^{-2}$ to $0.32 \AA^{-1}$ to be covered. After normalisation in respect to thickness, transmission and measuring time, the solvent signal was subtracted from the sample signal, and the raw intensities were converted to a scattering cross-section $I(q)$ on an absolute scale $\left(\mathrm{cm}^{-1}\right)$. All the present experiments were conducted at $25^{\circ} \mathrm{C}$.

SAXS data were analysed using the Zimm formalism described in Section 1. Figure 5 represents the reverse apparent mass as a function of asphaltene concentration (see $E q$. 7) for both feed and effluent asphaltenes. The increase in ordinate, significant in disaggregation, is clearly related to the change in slope from slightly positive values for feeds to largely negative values for effluents close to flocculation onset: the variations in the measured values $A_{2}$ and $M_{w}$ indicate during the hydroconversion process a progressive disaggregation and destabilisation of the colloidal suspension. This observation is related to the $\mathrm{H} / \mathrm{C}$ ratio decrease which is thought to occur mainly by the de-alkylation process.

These observations could be discussed in the framework of the Yen micelle [27] or the recently proposed nanoaggregate [28] based on formation of aggregates by $\pi-\pi$ interactions of fused aromatic rings which, as a consequence, have peripheral aliphatic or naphthenic moities. These peripheral aliphatic species could induce steric repulsion. The 
repulsion efficiency is in this case related to density and length of the peripheral chains; both of these parameters will change during conversion by the de-alkylation process. However, the simultaneous mass diminution on one hand, and tendency to aggregation, as revealed by $A_{2}$ decrease, on the other hand, could not be explained only by considering the attractive interaction between nano-aggregates: an additional interaction type should certainly be taken into account.

\section{CONCLUSION}

The robust method proposed to analyse small-angle scattering data enables one to extract meaningful parameters such as $R_{g}, M_{w}$ and $A_{2}$, characterising asphaltene aggregates.

Many recent studies succeeded in relating structure on a nanometre length scale to macroscopic properties. To summarise, we showed that $R_{g}, M_{w}$ and $A_{2}$ are mutually dependent through a single parameter: the mass fractal dimension $D_{f}$ of aggregates. The structural picture which emerges from this study enables one to predict viscosity of a solution at any given asphaltene concentration. The adsorption behaviour from asphaltene solution to water or a solid interface was successfully related to bulk aggregate characteristics. In particular, $R_{g}$ was found to be of the same order of magnitude as the thickness of the adsorbed layer as measured by SANS in contrast-matching conditions at water/solution interfaces or by neutron reflectivity at solid/solution interfaces. This observation strongly supports the adsorption of asphaltenes as a monolayer of aggregates. Moreover, the surface excess was found to be dependent on $M_{w}$, implying a densification confirmed independently by neutron measurements. Finally, the repulsive/attractive interaction balance between aggregates, as reflected by $A_{2}$ values, was related to the relative stability of water in oil emulsions and to the stability of hydro-conversion effluents. The single model, namely, the polydisperse mass fractal one, enables one to describe correctly different macroscopic properties such as viscosity, adsorption, relative stability of the emulsion and colloidal stability of hydro-conversion effluents.

From comparison of SAXS and SANS data a dimension smaller than $15 \AA$, representing the size of internal heterogeneity, was evidenced. This discrepancy observed at large $q$ values between $\mathrm{X}$-rays and neutrons rules out simple form factors based on homogeneous particles and opens the way to new questions:

What is the building block unit of such a structure? A "good" candidate for this unit is obviously the "nano-aggregate" [11] or the Yen micelle [27], whose heterogeneous nature (aromatic centre and aliphatic periphery) is consistent with the discrepancy observed between SAXS and SANS data at large $q$ values. This object was evidenced at small asphaltene concentration (a few tens of $\mathrm{mg} / \mathrm{l}$ ) and its dimensions, $\approx 10-20 \AA[17,18]$, mainly defined by its hydrodynamic properties, are in line with the contrast inhomogeneities smaller than $15 \AA$. Small-angle scattering experiments were performed on more concentrated solution (a few thousand $\mathrm{mg} / \mathrm{l})$ and give values of radius of gyration $(\approx 50$ to $100 \AA$ A ) consistent with hydrodynamic sizes recently measured by PFG NMR $[17,18]$ and higher than sizes of nanoaggregates. This is in favour of clusterisation of nano-aggregates in the range $100-1000 \mathrm{mg} / \mathrm{l}$. It is worth noting that this behaviour is also consistent with the hierarchical structure proposed by Yen.

The second unanswered question to assess the mass fractal structure concerns the nature of nano-aggregate interaction, and consequently the process that limits the aggregate size. A possible way to gain insight is to take advantage of enhanced contrast between asphaltene and deuterated toluene and high flux to run neutron experiments in the low concentration domain.

Most of the experiments presented in this paper concern model oils, i.e. asphaltene extracted from oil and dissolved in good solvents. Efforts to probe by the same robust approach real crude oils are highly desirable. If SANS is difficult to apply to such a system due to the high incoherent scattering cross-section of hydrogen, SAXS has the potential to clarify the structure of asphaltene aggregates in crude oils. Moreover, real conditions such as high temperature are reachable using this technique.

\section{ACKNOWLEDGEMENTS}

This article is the result of several different contributions, and in the first place we thank S. Charles, N. Jouault, Y. Corvis and J. Gummel for their active assistance during their stay at IFP Y. Briolant, L. Zupancic and J. M'Hamdi are warmly acknowledged for their help in conducting experiments. We are grateful to F. Cousin (LLB) for his involvement in neutron reflectivity and to D. Frot, D. Espinat and J. Verstraete for useful discussions. J. Eyssautier is acknowledged for language correction.

\section{REFERENCES}

1 Sanière A., Hénaut I., Argillier J.F. (2004) Pipeline Transportation of Heavy Oils, a Strategic, Economic and Technological Challenge, Oil Gas Sci. Technol. - Rev. IFP 59, $5,455-466$

2 Pierre C., Barré L., Pina A., Moan M. (2004) Composition and Heavy Oil Rheology, Oil Gas Sci. Technol. - Rev. IFP 59, 5 , 489-501.

3 Hemmingsen P.V., Silset A., Hannisdal A., Sjoblom J. (2005) Emulsions of Heavy Crude Oils I: Influence of Viscosity, Temperature and Dilution, J. Disper. Sci.Technol. 26, 615-627. 
4 Jestin J., Simon S., Zupancic L., Barré L. (2007) A Small Angle Neutron Scattering Study of the Adsorbed Asphaltene Layer in Water-in-Hydrocarbon Emulsions: Structural Description Related to Stability, Langmuir 23, 21, 10471-10478.

5 Nabzar L., Aguiléra M.E. (2008) The Colloidal Approach: A Promising Route for Asphaltene Deposition Modelling, Oil Gas Sci.Technol.-Rev. IFP 63, 1, 21-35.

6 Sinquin A., Palermo T., Peysson Y. (2004) Rheological and Flow Properties of Gas Hydrate Suspensions, Oil Gas Sci. Technol. - Rev. IFP 59, 41-57.

7 Speight J.G. (2004) Petroleum Asphaltenes - Part 1: Asphaltenes, Resins and the Structure of Petroleum, Oil Gas Sci. Technol. - Rev. IFP 59, 467-477.

8 Hortal A.R., Martínez-Haya B., Lobato M.D., Pedrosa J.M., Lago S. (2006) On the determination of molecular weight distributions of asphaltenes and their aggregates in laser desorption ionization experiments, J. Mass Spectrom . 41, 7, 960968.

9 Groenzin H., Mullins O.C. (1999) Asphaltene Molecular Size and Structure, J. Phys. Chem. A 103, 50, 11237-11245.

10 Groenzin H., Mullins O.C., Eser S., Mathews J., Yang M.G., Jones D. (2003) Molecular Size of Asphaltene Solubility Fractions, Energ. Fuel. 17, 2, 498-503.

11 Andreatta G., Goncalves C.C., Buffin G., Bostrom N., Quintella C.M., Arteaga-Larios F., Perez E., Mullins O.C. (2005) Nanoaggregates and Structure-Function Relations in Asphaltenes, Energ. Fuel. 19, 4, 1282-1289.

12 Goncalves S., Castillo J., Fernandez A., Hung J. (2004) Absorbance and fluorescence spectroscopy on the aggregation behavior of asphaltene-toluene solutions, Fuel 83, 13, 1823-1828.

13 Fenistein D., Barré L., Broseta D., Espinat D., Livet A., Roux J.N., Scarsella M. (1998) Viscosimetric and Neutron Scattering Study of Asphaltene Aggregates in Mixed Toluene/Heptane Solvents, Langmuir 14, 5, 1013-1020.

14 Espinat D. (1990) Application of Light, X-Ray and Neutron Diffusion Techniques to the Study of Colloidal Systems, Revue de l'Institut Français du Pétrole 45, 595-820.

15 Spiecker P.M., Gawrys K.L., Trail C.B., Kilpatrick P.K. (2003) Effects of petroleum resins on asphaltene aggregation and waterin-oil emulsion formation, Colloid. Surface. A 220, 1-3, 9-27.

16 Acevedo S., Gutierrez L.B., Negrin G., Pereira J.C., Mendez B., Delolme F., Dessalces G., Broseta D. (2005) Molecular Weight of Petroleum Asphaltenes: A Comparison between Mass Spectrometry and Vapor Pressure Osmometry, Energ. Fuel. 19, $4,1548-1560$.

17 Freed D., Lisitza N., Sen P., Song Y. (2007) Molecular Composition and Dynamics of Oils from Diffusion Measurements, in Asphaltene Heavy Oils and Petroleomics, Mullins O.C., Sheu E.Y., Hammami A., Marshall A.G. (eds.), Springer, New York, pp. 279-299.

18 Kawashima H., Takanohashi T., Iino M., Matsukawa S. (2008) Determining Asphaltene Aggregation in Solution from Diffusion Coefficients As Determined by Pulsed-Field Gradient Spin-Echo 1H NMR, Energ. Fuel. 22, 6, 3989-3993.

19 Fenistein D., Barré L., Frot D. (2000) From Aggregation to Flocculation of Asphaltenes, a Structural Description by Radiation Scattering Techniques, Oil Gas Sci. Technol. - Rev. IFP 55, 1, 123-128

20 Fenistein D., Barré L. (2001) Experimental measurement of the mass distribution of petroleum asphaltene aggregates using ultracentrifugation and small-angle X-ray scattering, Fuel 80, 2, 283-287.
21 Gawrys K.L., Kilpatrick P.K. (2005) Asphaltenic aggregates are polydisperse oblate cylinders, J. Colloid Interf. Sci. 288, 2, 325-334.

22 Gawrys K.L., Blankenship G.A., Kilpatrick P.K. (2006) Solvent Entrainment in and Flocculation of Asphaltenic Aggregates Probed by Small-Angle Neutron Scattering, Langmuir 22, 10, 4487-4497.

23 Sirota E.B. (2005) Physical Structure of Asphaltenes, Energ. Fuel. 19, 4, 1290-1296.

24 Sirota E.B., Lin M.Y. (2007) Physical Behavior of Asphaltenes, Energ. Fuel. 21, 5, 2809-2815.

25 Barré L., Simon S., Palermo T. (2008) Solution Properties of Asphaltenes, Langmuir 24, 3709-3717.

26 Barré L., Espinat D., Rosenberg E., Scarsella M. (1997) Colloidal Structure of Heavy Crudes and Asphaltene Solutions, Revue de l'Institut Français du Pétrole 52, 2, 161-175.

27 Yen T.F., Erdman J.G., Pollack S.S. (1961) Investigation of the Structure of Petroleum Asphaltenes by X-Ray Diffraction, Anal. Chem. 33, 11, 1587-1594.

28 Mullins O.C. (2007) Petroleomics and Structure Function Relations in Asphaltenes and Crude Oils, in Asphaltenes, Heavy Oils, and Petroleomics, Mullins O.C., E.Y.S., Hammami A., Marshall A.G. (eds.), Springer, New York, pp. 1-16.

29 Burchard W. (1999) Solution properties of branched macromolecules, Adv. Polym. Sci. 143, 113-194.

30 Roux J.N., Broseta D., Deme B. (2001) SANS Study of Asphaltene Aggregation: Concentration and Solvent Quality Effects, Langmuir 17, 16, 5085-5092.

31 Sheu E.Y., Liang K.S., Sinha S.K., Overfield R.E. (1992) Polydispersity analysis of asphaltene solutions in toluene, $J$. Colloid Interf. Sci. 153, 2, 399-410.

32 Marques J., Merdrignac I., Baudot A., Barré L., Guillaume D., Espinat D., Brunet S. (2008) Asphaltenes Size Polydispersity Reduction by Nano- and Ultrafiltration Separation Methods Comparison with the Flocculation Method, Oil Gas Sci. Technol. - Rev. IFP 63, 1, 139-149.

33 Mack C. (1932) Colloid chemistry of asphalts, J. Phys. Chem. 36, 2901-2914

34 Reerink H. (1973) Size and shape of asphaltene particles in relation to high-temperature viscosity, Ind. Eng. Chem. Product Res. Dev. 12, 11, 82-88.

35 Altgelt K.H., Harle O.L. (1975) Effect of asphaltenes on asphalt viscosity, Ind. Eng. Chem. Product Res. Dev. 14, 4, 240-246.

36 Einstein A. (1911) A New Determination of Molecular Dimensions, Annalen der Physik 34, 591-592.

37 Batchelor G.K., Green J.T. (1972) The hydrodynamic interaction of two small freely-moving spheres in a linear flow field, $J$. Fluid Mech. 56, 375-400.

38 Quemada D. (1977) Rheology of concentrated disperse systems and minimum energy dissipation principle, Rheol. Acta 16, 82-94.

39 Storm D.A., Sheu E.Y. (1993) Rheological studies of Ratawi vacuum residue at $366 \mathrm{~K}$, Fuel 72, 2, 233-237.

40 Buckley J.S., Liu Y. (1998) Some mechanisms of crude oil/brine/solid interactions, J. Petrol. Sci. Eng. 20, 3-4, 155-160.

41 Buckley J.S., Lord D.L. (2003) Wettability and morphology of mica surfaces after exposure to crude oil, J. Petrol. Sci. Eng. 39, 3-4, 261-273.

42 Dudasova D., Simon S., Hemmingsen P., Sjöblom J. (2008) Study of asphaltenes adsorption onto different minerals and clays. Part 1. Experimental adsorption with UV depletion detection, Colloid. Surface. A 317, 1-9. 
43 Pernyeszi T., Patzko A., Berkesi O., Dekany I. (1998) Asphaltene adsorption on clays and crude oil reservoir rocks, Colloid. Surface. A 137, 1-3, 373-384.

44 Gonzalez G., Middea A. (1987) Asphaltenes Adsorption by Quartz and Feldspar, J.Disper.Sci.Technol. 8, 5, 525-548.

45 Jouault N., Corvis Y., Cousin F., Jestin J., Barré L. (2009) Asphaltene adsorption mechanisms at the local scale probed by neutron reflectivity: transition from mono to multilayer growth above flocculation threshold, Langmuir 25, 3991-3998.

46 Simon S., Jestin J., Palermo T., Barré L. (2009) Relation between Solution and Interfacial Properties of Asphaltene Aggregates, Energ. Fuel. 23, 1, 306-313.

47 Bouriat P., El Kerri N., Graciaa A., Lachaise J. (2004) Properties of a Two-Dimensional Asphaltene Network at the Water/Cyclohexane Interface Deduced from Dynamic Tensiometry, Langmuir 20, 18, 7459-7464.

48 Yarranton H.W., Sztukowski D.M., Urrutia P. (2007) Effect of interfacial rheology on model emulsion coalescence: I. Interfacial rheology, J. Colloid Interf. Sci. 310, 1, 246-252.

49 Freer E.M., Radke C.J. (2004) Relaxation of Asphaltenes at the Toluene/Water Interface: Diffusion Exchange and Surface Rearrangement, J. Adhesion 80, 6, 481-496.

50 Stanford L.A., Rodgers R.P., Marshall A.G., Czarnecki J., Wu X.A. (2007) Compositional Characterization of Bitumen/Water Emulsion Films by Negative- and Positive-Ion Electrospray Ionization and Field Desorption/Ionization Fourier Transform Ion Cyclotron Resonance Mass Spectrometry, Energ. Fuel. 21, 2, 963-972.
51 Zhang L.Y., Breen P., Xu Z., Masliyah J.H. (2007) Asphaltene Films at a Toluene/Water Interface, Energ. Fuel. 21, 1, 274-285.

52 McLean J.D., Kilpatrick P.K. (1997) Effects of Asphaltene Aggregation in Model Heptane-Toluene Mixtures on Stability of Water-in-Oil Emulsions, J. Colloid Interf. Sci. 196, 1, 23-34.

53 McLean J.D., Kilpatrick P.K. (1997) Effects of Asphaltene Solvency on Stability of Water-in-Crude-Oil Emulsions, $J$. Colloid Interf. Sci. 189, 2, 242-253.

54 Anisimov M.A., Yudin I.K., Nikitin V., Nikolaenko G., Chernoutsan A., Toulhoat H., Frot D., Briolant Y. (1995) Asphaltene Aggregation in Hydrocarbon Solutions Studied by Photon Correlation Spectroscopy, J. Phys. Chem. 99, 23, 9576-9580.

55 Yudin I.K., Nikolaenko G.L., Gorodetskii E.E., Kosov V.I., Melikyan V.R., Markhashov E.L., Frot D., Briolant Y. (1998) Mechanisms of asphaltene aggregation in toluene-heptane mixtures, J. Petrol. Sci. Eng. 20, 3-4, 297-301.

56 Gummel J., Corvis Y., Jestin J., M'Hamdi J., Barré L. (2009) Asphaltene multilayer growth in porous medium probed by SANS, Eur. Phys. J. Special Topics 168, 171-176.

57 Merdrignac I., Quoineaud A.-A., Gauthier T. (2006) Evolution of Asphaltene Structure during Hydroconversion Conditions, Energ. Fuel. 20, 5, 2028-2036.

Final manuscript received in March 2009 Published online in September 2009 